\title{
The Macintosh as a user-friendly laboratory for perception and cognition
}

\author{
JAMSHED J. BHARUCHA, BLAKE MEIKE, and JOHN C. BAIRD \\ Dartmouth College, Hanover, New Hampshire
}

\begin{abstract}
We present a radically user-friendly Macintosh laboratory, MindLab, for instruction in perception and cognition. MindLab's forte is its ability to display pictorial stimuli, including digitized photographs, transferred via the clipboard from Macintosh graphics applications. An experiment is programmed by specifying sequences of event primitives, represented by icons, to which stimuli, temporal parameters, and feedback options are assigned.
\end{abstract}

Research and instruction in perception and cognition can be greatly enhanced by microcomputer-based laboratories that are radically user friendly. In this paper, we introduce MindLab, which is easily programmed to run a wide range of experiments involving the graphic display of static images.

Dartmouth has embarked on an effort to incorporate the Macintosh into the curriculum (see Levine, Strenta, \& Wolford, 1986). Approximately $75 \%$ of incoming students purchase Macintoshes, and the remaining $25 \%$ have easy access to Macintoshes all over campus. Dormitory rooms and classrooms are hard wired to the mainframe time-sharing systems to permit communication between micros and mainframes, and classrooms are equipped with facilities to either project the Macintosh image onto a large screen or display it on multiple video monitors.

Among the many pedagogical opportunities afforded by such an environment (see Chute, 1986; Hewett, 1986; McCord, 1986; Perkey, 1986) is a substantial laboratory component in even the most elementary of courses. MindLab permits students to program and carry out experiments that would ordinarily be beyond the scope of all but laboratory courses. Easy access to a network linking micros via mainframes permits highly distributed data collection. An instructor can make experiments available over the network. As an assignment, students can run themselves as subjects in their own rooms and can send the data files to the instructor's account. For student projects, experiments programmed by students can be made available to the class over the network, and the data files can be mailed to the students' accounts.

This work was supported by a grant from the Keck Foundation. The authors are grateful to George Wolford and Steven Maker for valuable suggestions.

MacWrite, Edit, MacPaint, MacDraw, and Switcher are trademarks of Apple Computer. Multiplan, CheapPaint, SuperPaint, and ThunderScan are trademarks of Microsoft, MicroMind, Silicon Beach Software, and Thunderware, respectively.

MindLab was developed on the Lisa development system using Lisa Pascal and MacApp. It will be released through Academic Courseware Exchange, Kinko's Service Corporation, 4141 State St., Santa Barbara, CA 93110. Requests for reprints of this paper should be sent to Jamshed J. Bharucha, Department of Psychology, Dartmouth College, Hanover, NH 03755.

\section{DEVELOPMENT GOALS}

MindLab was developed to meet several criteria.

(1) It presumes no prior programming knowledge. The only prerequisite is a familiarity with the Macintosh (including menus, windows, icons, selecting, deselecting, opening, closing, and dragging) and a familiarity with a graphics application, such as MacPaint.

(2) MindLab requires a minimal learning period. This is important in order not to overwhelm the instructional purpose, which should be focused on the psychological issues and on experimental design, not on implementation.

(3) MindLab is generative, allowing as wide a range of designs as possible with a very small number of primitive events. An interstimulus interval is implemented as a blank stimulus presented for a specified period of time. If the subject is to control the onset of each trial, MindLab is programmed to expect a response, which it records as though it were a response to be measured; the experimenter simply ignores the data stored for that response. Finally, an experiment consisting of a study phase and a test phase is implemented as two MindLab documents, the first of which may involve no response.

(4) A MindLab experiment can be decomposed into its constituent trials. The experimenter can thus preview trials individually without having to run through the entire sequence.

(5) The construction of a MindLab experiment is highly interactive. The experimenter can test and run himself/ herself on selected trials and examine the data immediately.

(6) MindLab requires no peripheral devices. This is equivalent to Hewett's (1986) criterion of transportability; courseware is transportable if it is "usable wherever the student has a machine to use, not just in an instructional laboratory" (p. 190).

(7) MindLab exploits the graphics capabilities of the Macintosh and takes advantage of the growing number and sophistication of graphics packages. The program's strength is the presentation of pictorial stimuli; text is considered a special case of pictorial stimuli.

(8) MindLab is multifunctional; that is, it is "usable 


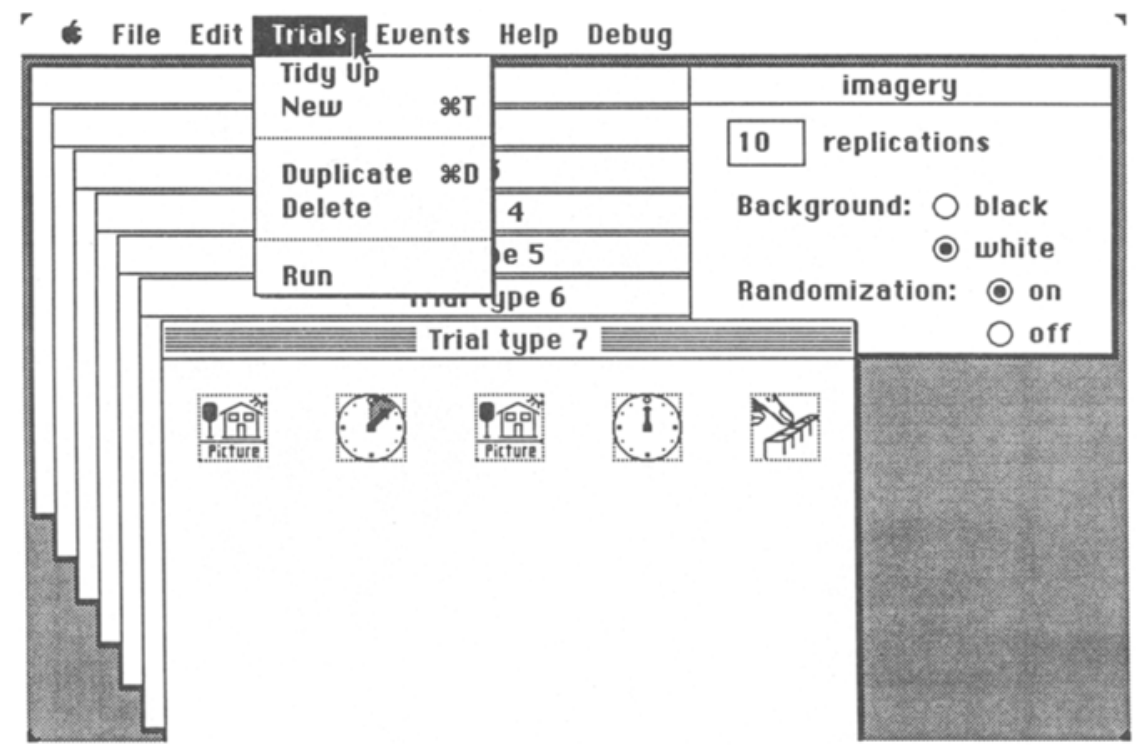

Figure 1. A window is created for each uniquely different trial type. The number of identical replications, the background, and the randomization of trials are specified in the window in the top right corner.

in several ways or in more than one course (e.g., as a demonstration in introductory psychology and as an experiment simulator in one or more upper-level courses)" (Hewett, 1986, p. 190).

\section{RUN MODES}

MindLab runs in two modes: subject mode and experimenter mode. Subject mode prompts for an output file name and a subject identification number. The subject clicks on "Run" to begin. Experimenter mode is used to construct, preview, and test the experiment.

\section{TRIALS AND TRIAL TYPES}

A MindLab experiment is constructed by specifying each uniquely different trial, called a trial type. The experimenter creates a window for each trial type and inserts event icons representing the sequence of events in that trial type. The number of identical replications of each trial type is entered in the experiment window at the top right of the screen (see Figure 1). If the randomization option in the experiment window is turned on, trials are presented to the subject in random order, with a new randomization for each replication and each subject.

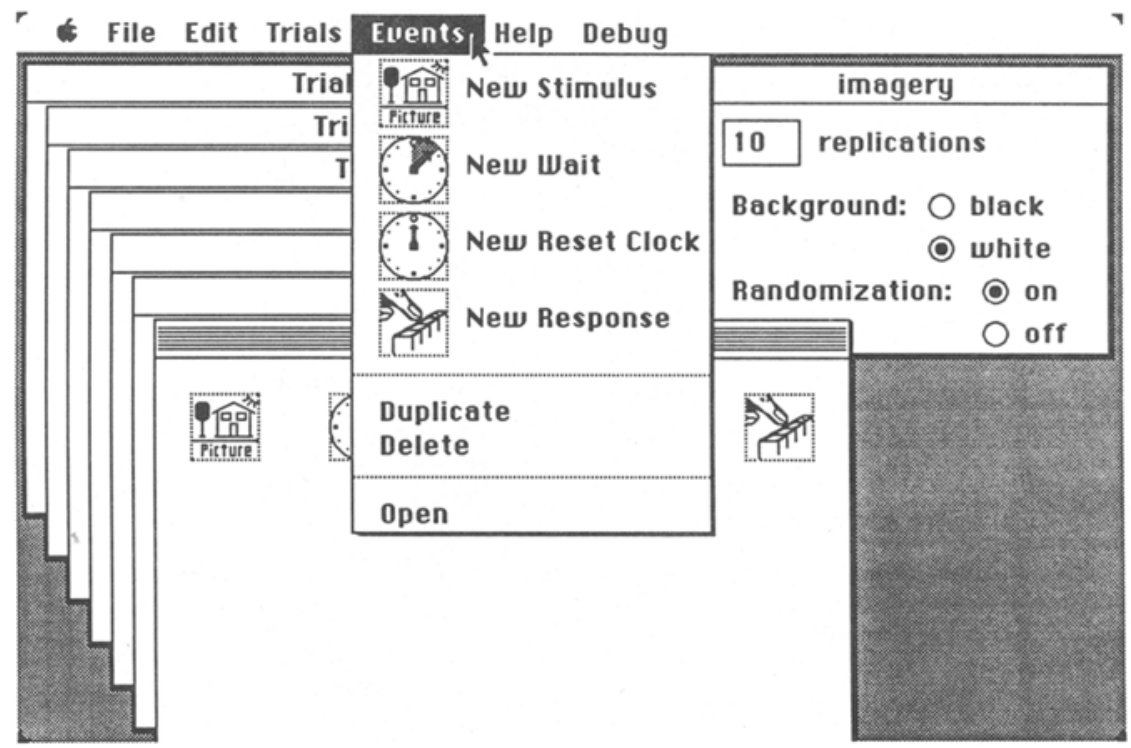

Figure 2. Four types of event icons constitute the primitives for specifying the sequence of events in a trial. 


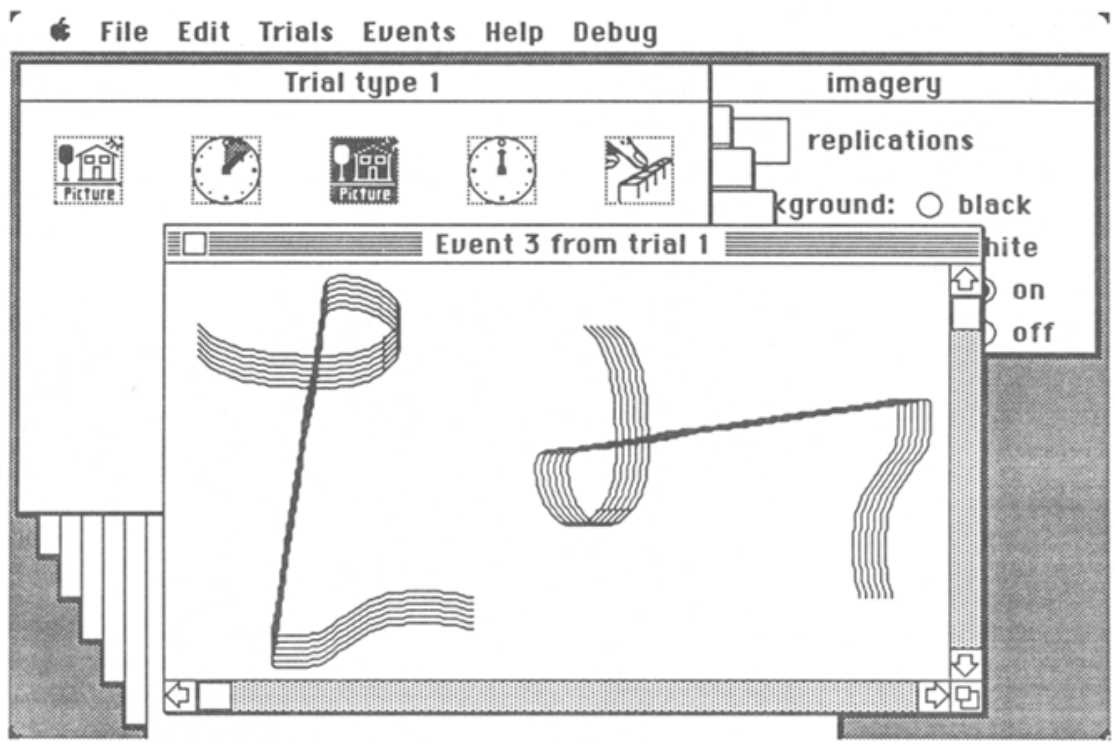

Figure 3. Stimulus icons are assigned an image by pasting from the clipboard. The stimulus then can be examined by opening the icon into a window. In this example, the stimulus is a pair of figures in a mental rotation task adapted from Shepard and Metzler (1971). Event 1 presents a blank screen for a duration specified by Event 2 . Event 3 presents the figures to be compared. Event 4 starts the clock, and Event 5 waits for and records the subject's response.

A trial type window can be selected (for editing or previewing) either by clicking on the window or from a scrollable menu.

A trial type may be duplicated to create another trial type, whose icon sequence can then be edited or whose icons can be assigned different stimuli or temporal parameters.

\section{EVENTS}

There are four event primitives-stimulus, wait, reset clock, and response-represented by icons (see Figure 2). A stimulus icon presents a pre-stored image. A wait icon waits for a specified length of time. A response icon waits for and records a keyboard response and the time elapsed since the last clock reset. Each trial type can accommodate up to 15 event icons, which can be arranged in any order, including multiple stimuli and responses. Event icons can be rearranged within a window by dragging.

A new stimulus or wait icon displays a question mark until loaded with the necessary information. A stimulus is assigned to a stimulus icon by selecting it and pasting an image from the clipboard. The stimulus assigned to a stimulus icon can be viewed by opening the icon, as in Figure 3. Wait time is specified in a window opened from the wait icon.

\section{STIMULUS GENERATION}

A stimulus can be any image copied to the clipboard from a Macintosh graphics application, such as MacPaint, MacDraw, SuperPaint, CheapPaint (a desk accessory), or ThunderScan. Since the clipboard can store only one image at a time, stimulus assignment can be facilitated by the Scrapbook file (see Figure 4), which can store a number of images, and/or Switcher, which partitions the Macintosh's memory and permits switching between MindLab and a graphics package.

Experiments involving the controlled presentation of photographs or the editing of photographs are easily implemented by digitizing the photographs (using ThunderScan, for instance), provided that the resulting reduction in resolution is acceptable.

MindLab stores images in PICT format, the interpretation of which may be slow and variable for pictures generated by graphics packages that are object oriented (e.g., MacDraw). In future versions of MindLab, the interpretation and expansion of the PICT representation into a bitmap will anticipate the stimulus onset. At present, variability in interpretation time can be eliminated by transforming object-oriented images into bitmaps via MacPaint before pasting to a stimulus icon.

MindLab centers the image on the screen. For large experiments, storage requirements can be reduced by clipping a frame around the desired stimulus rather than clipping the entire graphics document. The background (black or white) to the clipped frame can be set using the experiment window.

\section{FEEDBACK}

Two kinds of optional feedback can be given to the subject after each response: response time and correct/incorrect. For correct/incorrect feedback, the experimenter 


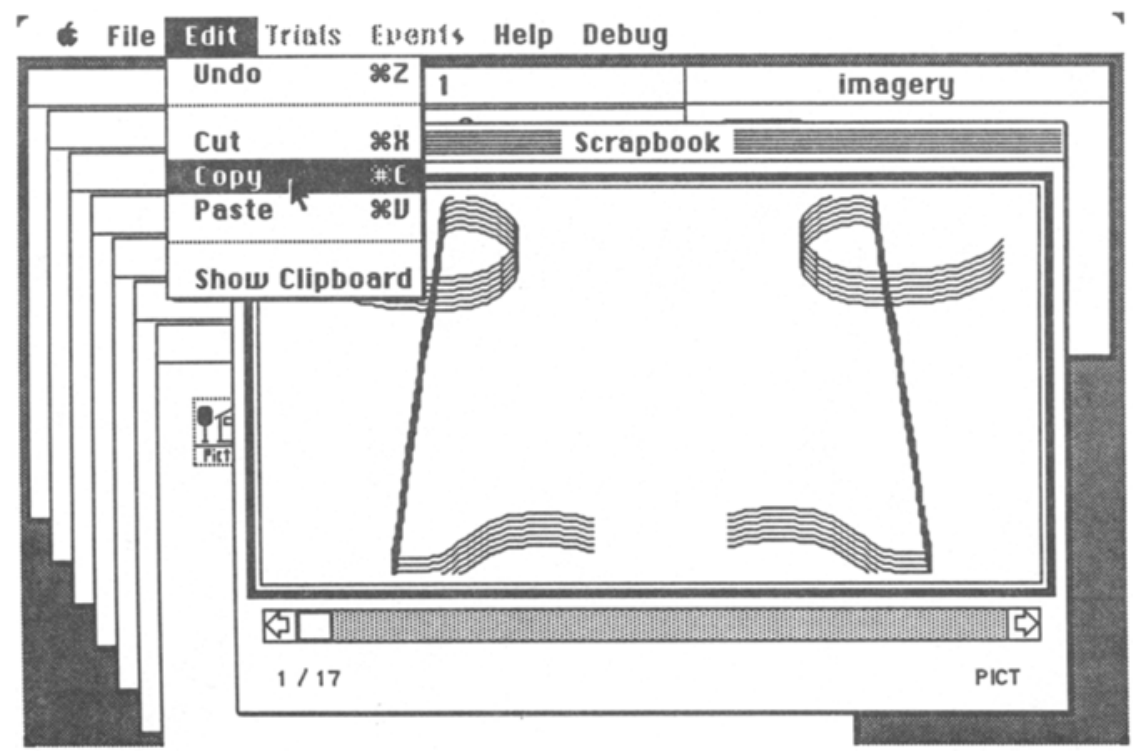

Figure 4. Stimuli can be copied from the scrapbook file, which can be loaded in advance from a graphics package.

specifies, in a window that appears by opening a response icon, the keyboard character that counts as correct.

\section{EXPERIMENTER PREVIEWS}

The experimenter can preview a selected trial type or all trial types in sequence by selecting "Run" from the Trial menu or the File menu. A trial type cannot be previewed if any of its icons display a question mark. The data from experimenter previews are displayed in a data window.

\section{DATA}

When in subject mode, the data from a run are stored in a TEXT file, which contains a record for each response collected. Records are delimited by carriage returns. Each record contains the following fields, delimited by tabs: subject identification number, trial sequence number, trial type, the ASCII code for the key pressed, and response time. TEXT files can therefore be read by many statistical programs available for the Macintosh (see Lehman, 1986) or can be transferred to mainframes for input to larger packages. Sorting or reformatting can be accomplished via editors, such as MacWrite or Edit, or programs with data-manipulation capabilities, such as Multiplan.

\section{REFERENCES}

Chute, D. L. (1986). MacLaboratory for psychology: General experimental psychology with Apple's Macintosh. Behavior Research Methods, Instruments, \& Computers, 18, 205-209.

HewETT, T. T. (1986). When every student has a computer: A new perspective on courseware and its development. Behavior Research Methods, Instruments, \& Computers, 18, 188-195.

Lehman, R. S. (1986). Macintosh statistical packages. Behavior Research Methods, Instruments, \& Computers, 18, 177-187.

Levine, L., Strenta, C., \& Wolford, G. (1986). The impact of the Macintosh at Dartmouth (1984/1985). Hanover, NH: Dartmouth College.

McCoRD, J. (1986). Computing: Is it a better mousetrap? Behavior Research Methods, Instruments, \& Computers, 18, 210-213.

PERKEY, M. N. (1986). The effect of a machine-rich environment on courseware development: The process and the product. Behavior Research Methods, Instruments, \& Computers, 18, 196-204.

SHEPARD, R. N., \& METZLER, J. (1971). Mental rotation of three dimensional objects. Science, 171, 701-703. 